\title{
Integral inequalities via generalized quasiconvexity with applications
}

Eze R. Nwaeze ${ }^{1 *}$

\section{*Correspondence:}

enwaeze@alasu.edu

${ }^{1}$ Department of Mathematics and

Computer Science, Alabama State

University, Montgomery, USA

\begin{abstract}
Two classes of functions are hereby considered; namely, $\eta$-quasiconvex, and strongly $\eta$-quasiconvex functions. For the former, we establish some novel integral inequalities of the trapezoid kind for functions with second derivatives, while, for the latter, we obtain some new estimates of the integral $\int_{\alpha}^{\beta}(\mathfrak{r}-\alpha)^{p}(\beta-\mathfrak{r})^{a} \mathcal{K}(\mathfrak{r}) d \mathfrak{r}$ when $|\mathcal{K}(\mathfrak{r})|$, to some powers, is strongly $\eta$-quasiconvex. Results obtained herein contribute to the development of these new classes of functions by providing broader generalizations to some well-known results in the literature. Furthermore, we employ our results to deduce some estimates for the perturbed version of the trapezoidal formula. Finally, applications to some special means are also presented.
\end{abstract}

MSC: 26A51;26D15; 26E60;41A55

Keywords: Hermite-Hadamard inequality; Convex functions; Quasiconvex functions; Trapezoidal formula; Special means

\section{Introduction}

Nearly no mathematician can ignore the significant role of convex sets and convex functions in applied mathematics, especially in nonlinear programming and optimization theory. In addition, the elegance of convex sets and functions in shape and property makes studying this branch of mathematical analysis attractive. On the other hand, it should be observed that in new problems related to convexity, generalized notions for convex sets and functions are required to reach favorite and applicable results. In the past six decades, many determined attempts have gone in universalizing the concept of convexity. One of such generalizations is the concept of quasiconvex functions given in the definition below.

Unless otherwise stated, we shall assume throughout this paper that $\mathfrak{D} \subseteq \mathbb{R}$ is an interval, and $\mathfrak{D}^{\circ}$ represents the interior of $\mathfrak{D}$.

Definition 1 ([6]) A function $\mathcal{K}: \mathfrak{D} \rightarrow \mathbb{R}$ is called quasiconvex on $\mathfrak{D}$ if

$$
\mathcal{K}(\tau \mathfrak{u}+(1-\tau) \mathfrak{v}) \leq \max \{\mathcal{K}(\mathfrak{u}), \mathcal{K}(\mathfrak{v})\}
$$

for all $\mathfrak{u}, \mathfrak{v} \in \mathfrak{D}$ and $\tau \in[0,1]$

For this class of quasiconvex functions, Ion established the following results.

(c) The Author(s) 2019. This article is distributed under the terms of the Creative Commons Attribution 4.0 International License (http://creativecommons.org/licenses/by/4.0/), which permits unrestricted use, distribution, and reproduction in any medium, provided you give appropriate credit to the original author(s) and the source, provide a link to the Creative Commons license, and indicate if changes were made. 
Theorem 2 ([11]) Let $\mathcal{K}: \mathfrak{D} \rightarrow \mathbb{R}$ be a differentiable function on $\mathfrak{D}^{\circ}, \alpha, \beta \in \mathfrak{D}^{\circ}$ with $\alpha<\beta$. If $\left|\mathcal{K}^{\prime}\right|$ is quasiconvex on $[\alpha, \beta]$, then

$$
\left|\frac{\mathcal{K}(\alpha)+\mathcal{K}(\beta)}{2}-\frac{1}{\beta-\alpha} \int_{\alpha}^{\beta} \mathcal{K}(\mathfrak{r}) d \mathfrak{r}\right| \leq \frac{\beta-\alpha}{4} \max \left\{\left|\mathcal{K}^{\prime}(\alpha)\right|,\left|\mathcal{K}^{\prime}(\beta)\right|\right\} .
$$

Theorem 3 ([11]) Let $\mathcal{K}: \mathfrak{D} \rightarrow \mathbb{R}$ be a differentiable function on $\mathfrak{D}^{\circ}, \alpha, \beta \in \mathfrak{D}^{\circ}$ with $\alpha<\beta$. If $\left|\mathcal{K}^{\prime}\right|^{p /(p-1)}$ is quasiconvex on $[\alpha, \beta]$ for $p>1$, then

$$
\begin{aligned}
& \left|\frac{\mathcal{K}(\alpha)+\mathcal{K}(\beta)}{2}-\frac{1}{\beta-\alpha} \int_{\alpha}^{\beta} \mathcal{K}(\mathfrak{r}) d \mathfrak{r}\right| \\
& \quad \leq \frac{\beta-\alpha}{2(p+1)^{1 / p}}\left[\max \left\{\left|\mathcal{K}^{\prime}(\alpha)\right|^{p /(p-1)},\left|\mathcal{K}^{\prime}(\beta)\right|^{p /(p-1)}\right\}\right]^{(p-1) / p} .
\end{aligned}
$$

Following the same line of thought, Alomari et al. proved the following.

Theorem 4 ([1]) Let $\mathcal{K}: \mathfrak{D} \rightarrow \mathbb{R}$ be a differentiable function on $\mathfrak{D}^{\circ}, \alpha, \beta \in \mathfrak{D}^{\circ}$ with $\alpha<\beta$. If $\left|\mathcal{K}^{\prime}\right|^{q}$ is quasiconvex on $[\alpha, \beta]$ for $q \geq 1$, then

$$
\left|\frac{\mathcal{K}(\alpha)+\mathcal{K}(\beta)}{2}-\frac{1}{\beta-\alpha} \int_{\alpha}^{\beta} \mathcal{K}(\mathfrak{r}) d \mathfrak{r}\right| \leq \frac{\beta-\alpha}{4}\left[\max \left\{\left|\mathcal{K}^{\prime}(\alpha)\right|^{q},\left|\mathcal{K}^{\prime}(\beta)\right|^{q}\right\}\right]^{1 / q} .
$$

In another direction, Özdemir et al. obtained an estimate for the integral $\int_{\alpha}^{\beta}(\mathfrak{r}-\alpha)^{p}(\beta-$ $\mathfrak{r})^{q} \mathcal{K}(\mathfrak{r}) d \mathfrak{r}$ in the following theorem.

Theorem 5 ([22]) Let $\mathcal{K}:[\alpha, \beta] \subset[0, \infty) \rightarrow \mathbb{R}$ be continuous on $[\alpha, \beta]$ such that $\mathcal{K} \in$ $L([\alpha, \beta])$ with $\alpha<\beta$. If $\mathcal{K}$ is quasiconvex on $[\alpha, \beta]$, then, for some fixed $p, q>0$, we obtain

$$
\begin{aligned}
& \int_{\alpha}^{\beta}(\mathfrak{r}-\alpha)^{p}(\beta-\mathfrak{r})^{q} \mathcal{K}(\mathfrak{r}) d \mathfrak{r} \\
& \quad \leq(\beta-\alpha)^{p+q+1} \mathcal{B}(p+1, q+1) \max \{\mathcal{K}(\alpha), \mathcal{K}(\beta)\},
\end{aligned}
$$

where $\mathcal{B}(\cdot, \cdot)$ is the Beta function defined for any $m, n>0$ as thus:

$$
\mathcal{B}(m, n)=\int_{0}^{1} \mathfrak{u}^{m-1}(1-\mathfrak{u})^{n-1} d \mathfrak{u} .
$$

Motivated by the above results, Liu proved the following results in same direction.

Theorem $6([16])$ Let $\mathcal{K}:[\alpha, \beta] \subset[0, \infty) \rightarrow \mathbb{R}$ be continuous on $[\alpha, \beta]$ such that $\mathcal{K} \in$ $L([\alpha, \beta])$ with $\alpha<\beta$, and let $x>1$ with $1 / x+1 / y=1$. If $|\mathcal{K}|^{y}$ is quasiconvex on $[\alpha, \beta]$, then, for some fixed $p, q>0$, we obtain

$$
\begin{aligned}
& \int_{\alpha}^{\beta}(\mathfrak{r}-\alpha)^{p}(\beta-\mathfrak{r})^{q} \mathcal{K}(\mathfrak{r}) d \mathfrak{r} \\
& \quad \leq(\beta-\alpha)^{p+q+1}[\mathcal{B}(p x+1, q x+1)]^{\frac{1}{x}}\left[\max \left\{|\mathcal{K}(\alpha)|^{y},|\mathcal{K}(\beta)|^{y}\right\}\right]^{\frac{1}{y}} .
\end{aligned}
$$

Theorem 7 Let $\mathcal{K}:[\alpha, \beta] \subset[0, \infty) \rightarrow \mathbb{R}$ be continuous on $[\alpha, \beta]$ such that $\mathcal{K} \in L([\alpha, \beta])$ with $\alpha<\beta$, and let $s \geq 1$. If $|\mathcal{K}|^{s}$ is quasiconvex on $[\alpha, \beta]$, then, for some fixed $p, q>0$, we 
obtain

$$
\begin{aligned}
& \int_{\alpha}^{\beta}(\mathfrak{r}-\alpha)^{p}(\beta-\mathfrak{r})^{q} \mathcal{K}(\mathfrak{r}) d \mathfrak{r} \\
& \quad \leq(\beta-\alpha)^{p+q+1} \mathcal{B}(p+1, q+1)\left[\max \left\{|\mathcal{K}(\alpha)|^{s},|\mathcal{K}(\beta)|^{s}\right\}\right]^{\frac{1}{s}} .
\end{aligned}
$$

Recently, Gordji et al. introduced a new class of convexity-called $\eta$-quasiconvex functions.

Definition 8 ([6]) A function $\mathcal{K}: \mathfrak{D} \rightarrow \mathbb{R}$ is called $\eta$-quasiconvex on $\mathfrak{D}$ with respect to $\eta: \mathbb{R} \times \mathbb{R} \rightarrow \mathbb{R}$ if

$$
\mathcal{K}(\tau \mathfrak{u}+(1-\tau) \mathfrak{v}) \leq \max \{\mathcal{K}(\mathfrak{v}), \mathcal{K}(\mathfrak{v})+\eta(\mathcal{K}(\mathfrak{u}), \mathcal{K}(\mathfrak{v}))\}
$$

for all $\mathfrak{u}, \mathfrak{v} \in \mathfrak{D}$ and $\tau \in[0,1]$.

A generalization of the above definition was recently proposed by Awan et al. as follows.

Definition 9 ([2]) A function $\mathcal{K}: \mathfrak{D} \rightarrow \mathbb{R}$ is called strongly $\eta$-quasiconvex on $\mathfrak{D}$ with respect to $\eta: \mathbb{R} \times \mathbb{R} \rightarrow \mathbb{R}$ and nonnegative modulus $\mu$ if

$$
\mathcal{K}(\tau \mathfrak{u}+(1-\tau) \mathfrak{v}) \leq \max \{\mathcal{K}(\mathfrak{v}), \mathcal{K}(\mathfrak{v})+\eta(\mathcal{K}(\mathfrak{u}), \mathcal{K}(\mathfrak{v}))\}-\mu \tau(1-\tau)(\mathfrak{v}-\mathfrak{u})^{2}
$$

for all $\mathfrak{u}, \mathfrak{v} \in \mathfrak{D}$ and $\tau \in[0,1]$.

Some papers concerning functions that are $\eta$-quasiconvex and strongly $\eta$-quasiconvex have been published. We refer the interested reader to $[4,5,7,8,13,17-19,21]$ and the references cited therein.

Inspired by the above results, it is our goal, in this present article, to:

1. Establish some perturbed inequalities akin to (1)-(3) for functions that are $\eta$-quasiconvex in absolute value. Three theorems are obtained in this direction and they extend Theorems 2-4 to this new class of functions. Applications of these results are also presented.

2. Extend Theorems 5-7 to the class of strongly $\eta$-quasiconvex functions. As a special case, our results boil down to already established theorems stated in this article. See Remark 19.

We arrange this article in the following manner: in Sect. 2, we state and prove our main results. Sections 3 and 4 present applications to the trapezoidal formula and special means, respectively.

\section{Main results}

For the proof of our main results, the following lemmas will be useful.

Lemma 10 ([23]) Let $\mathcal{K}: \mathfrak{D} \rightarrow \mathbb{R}$ be a differentiable function on $\mathfrak{D}^{\circ}, \alpha, \beta \in \mathfrak{D}^{\circ}$ with $\alpha \leq \beta$. If $\mathcal{K}^{\prime \prime} \in L([\alpha, \beta])$, then the following equality holds:

$$
\begin{aligned}
& \int_{\alpha}^{\beta} \mathcal{K}(\mathfrak{r}) d \mathfrak{r}-\frac{1}{2}(\beta-\alpha)(\mathcal{K}(\alpha)+\mathcal{K}(\beta))+\frac{5}{4}(\beta-\alpha)^{2}\left(\mathcal{K}^{\prime}(\beta)-\mathcal{K}^{\prime}(\alpha)\right) \\
& \quad=\frac{(\beta-\alpha)^{3}}{4} \int_{0}^{1}(\tau+1)^{2}\left[\mathcal{K}^{\prime \prime}(\tau \alpha+(1-\tau) \beta)+\mathcal{K}^{\prime \prime}(\tau \beta+(1-\tau) \alpha)\right] d \tau .
\end{aligned}
$$


Lemma $11([22])$ Let $\mathcal{K}:[\alpha, \beta] \subset[0, \infty) \rightarrow \mathbb{R}$ be continuous on $[\alpha, \beta]$ such that $\mathcal{K} \in$ $L([\alpha, \beta])$ with $\alpha<\beta$. Then for some fixed $p, q>0$ the following equality holds:

$$
\int_{\alpha}^{\beta}(\mathfrak{r}-\alpha)^{p}(\beta-\mathfrak{r})^{q} \mathcal{K}(\mathfrak{r}) d \mathfrak{r}=(\beta-\alpha)^{p+q+1} \int_{0}^{1}(1-\tau)^{p} \tau^{q} \mathcal{K}(\tau \alpha+(1-\tau) \beta) d \tau .
$$

\subsection{Perturbed trapezoid inequalities via $\eta$-quasiconvexity}

We now frame and justify our results for the class of $\eta$-quasiconvex functions.

Theorem 12 Let $\mathcal{K}: \mathfrak{D} \subset[0, \infty) \rightarrow \mathbb{R}$ be a differentiable function on $\mathfrak{D}^{\circ}$ such that $\mathcal{K}^{\prime \prime} \in$ $L([\alpha, \beta])$, where $\alpha, \beta \in \mathfrak{D}$ with $\alpha<\beta$. If $\left|\mathcal{K}^{\prime \prime}\right|$ is an $\eta$-quasiconvex function on $[\alpha, \beta]$, then

$$
\begin{aligned}
& \left|\int_{\alpha}^{\beta} \mathcal{K}(\mathfrak{r}) d \mathfrak{r}-\frac{1}{2}(\beta-\alpha)(\mathcal{K}(\alpha)+\mathcal{K}(\beta))+\frac{5}{4}(\beta-\alpha)^{2}\left(\mathcal{K}^{\prime}(\beta)-\mathcal{K}^{\prime}(\alpha)\right)\right| \\
& \quad \leq \frac{7(\beta-\alpha)^{3}}{12}\left(\mathbf{M}_{\alpha}^{\beta}\left(\left|\mathcal{K}^{\prime \prime}\right|, \eta\right)+\mathbf{N}_{\alpha}^{\beta}\left(\left|\mathcal{K}^{\prime \prime}\right|, \eta\right)\right),
\end{aligned}
$$

where $\mathbf{M}_{\alpha}^{\beta}\left(\left|\mathcal{K}^{\prime \prime}\right|, \eta\right)$ and $\mathbf{N}_{\alpha}^{\beta}\left(\left|\mathcal{K}^{\prime \prime}\right|, \eta\right)$ are defined by (10) and (11), respectively.

Proof By using the definition of $\eta$-quasiconvexity of $\left|\mathcal{K}^{\prime \prime}\right|$ on $[\alpha, \beta]$, one obtains the following inequalities:

$$
\begin{aligned}
\left|\mathcal{K}^{\prime \prime}(\tau \alpha+(1-\tau) \beta)\right| & \leq \max \left\{\left|\mathcal{K}^{\prime \prime}(\beta)\right|,\left|\mathcal{K}^{\prime \prime}(\beta)\right|+\eta\left(\left|\mathcal{K}^{\prime \prime}(\alpha)\right|,\left|\mathcal{K}^{\prime \prime}(\beta)\right|\right)\right\} \\
& =: \mathbf{M}_{\alpha}^{\beta}\left(\left|\mathcal{K}^{\prime \prime}\right|, \eta\right)
\end{aligned}
$$

and

$$
\begin{aligned}
\left|\mathcal{K}^{\prime \prime}(\tau \beta+(1-\tau) \alpha)\right| & \leq \max \left\{\left|\mathcal{K}^{\prime \prime}(\alpha)\right|,\left|\mathcal{K}^{\prime \prime}(\alpha)\right|+\eta\left(\left|\mathcal{K}^{\prime \prime}(\beta)\right|,\left|\mathcal{K}^{\prime \prime}(\alpha)\right|\right)\right\} \\
& =: \mathbf{N}_{\alpha}^{\beta}\left(\left|\mathcal{K}^{\prime \prime}\right|, \eta\right)
\end{aligned}
$$

for $\tau \in[0,1]$. Now, applying Lemma 10, and inequalities (10) and (11), we get

$$
\begin{aligned}
& \left|\int_{\alpha}^{\beta} \mathcal{K}(\mathfrak{r}) d \mathfrak{r}-\frac{1}{2}(\beta-\alpha)(\mathcal{K}(\alpha)+\mathcal{K}(\beta))+\frac{5}{4}(\beta-\alpha)^{2}\left(\mathcal{K}^{\prime}(\beta)-\mathcal{K}^{\prime}(\alpha)\right)\right| \\
& \quad \leq \frac{(\beta-\alpha)^{3}}{4} \int_{0}^{1}(\tau+1)^{2}\left|\mathcal{K}^{\prime \prime}(\tau \alpha+(1-\tau) \beta)+\mathcal{K}^{\prime \prime}(\tau \beta+(1-\tau) \alpha)\right| d \tau \\
& \quad \leq \frac{(\beta-\alpha)^{3}}{4} \int_{0}^{1}(\tau+1)^{2}\left[\left|\mathcal{K}^{\prime \prime}(\tau \alpha+(1-\tau) \beta)\right|+\left|\mathcal{K}^{\prime \prime}(\tau \beta+(1-\tau) \alpha)\right|\right] d \tau \\
& \quad=\frac{(\beta-\alpha)^{3}}{4}\left(\mathbf{M}_{\alpha}^{\beta}\left(\left|\mathcal{K}^{\prime \prime}\right|, \eta\right)+\mathbf{N}_{\alpha}^{\beta}\left(\left|\mathcal{K}^{\prime \prime}\right|, \eta\right)\right) \int_{0}^{1}(\tau+1)^{2} d \tau \\
& \quad=\frac{7(\beta-\alpha)^{3}}{12}\left(\mathbf{M}_{\alpha}^{\beta}\left(\left|\mathcal{K}^{\prime \prime}\right|, \eta\right)+\mathbf{N}_{\alpha}^{\beta}\left(\left|\mathcal{K}^{\prime \prime}\right|, \eta\right)\right) .
\end{aligned}
$$

That completes the proof of Theorem 12.

Theorem 13 Let $\mathcal{K}: \mathfrak{D} \subset[0, \infty) \rightarrow \mathbb{R}$ be a differentiable function on $\mathfrak{D}^{\circ}$ such that $\mathcal{K}^{\prime \prime} \in$ $L([\alpha, \beta])$, where $\alpha, \beta \in \mathfrak{D}$ with $\alpha<\beta$, and let $p>1$ with $1 / p+1 / q=1$. If $\left|\mathcal{K}^{\prime \prime}\right|^{q}$ is an $\eta$ - 
quasiconvex function on $[\alpha, \beta]$, then

$$
\begin{aligned}
& \left|\int_{\alpha}^{\beta} \mathcal{K}(\mathfrak{r}) d \mathfrak{r}-\frac{1}{2}(\beta-\alpha)(\mathcal{K}(\alpha)+\mathcal{K}(\beta))+\frac{5}{4}(\beta-\alpha)^{2}\left(\mathcal{K}^{\prime}(\beta)-\mathcal{K}^{\prime}(\alpha)\right)\right| \\
& \quad \leq \frac{(\beta-\alpha)^{3}}{4}\left(\frac{2^{2 p+1}-1}{2 p+1}\right)^{\frac{1}{p}}\left[\left(\mathbf{M}_{\alpha}^{\beta}\left(\left|\mathcal{K}^{\prime \prime}\right|^{q}, \eta\right)\right)^{\frac{1}{q}}+\left(\mathbf{N}_{\alpha}^{\beta}\left(\left|\mathcal{K}^{\prime \prime}\right|^{q}, \eta\right)\right)^{\frac{1}{q}}\right]
\end{aligned}
$$

where $\mathbf{M}_{\alpha}^{\beta}\left(\left|\mathcal{K}^{\prime \prime}\right|^{q}, \eta\right)$ and $\mathbf{N}_{\alpha}^{\beta}\left(\left|\mathcal{K}^{\prime \prime}\right|^{q}, \eta\right)$ are defined by (12) and (13), respectively.

Proof The $\eta$-quasiconvexity of the function $\left|\mathcal{K}^{\prime \prime}\right|^{q}$ implies that, for $\tau \in[0,1]$,

$$
\begin{aligned}
\left|\mathcal{K}^{\prime \prime}(\tau \alpha+(1-\tau) \beta)\right|^{q} & \leq \max \left\{\left|\mathcal{K}^{\prime \prime}(\beta)\right|^{q},\left|\mathcal{K}^{\prime \prime}(\beta)\right|^{q}+\eta\left(\left|\mathcal{K}^{\prime \prime}(\alpha)\right|^{q},\left|\mathcal{K}^{\prime \prime}(\beta)\right|^{q}\right)\right\} \\
& =: \mathbf{M}_{\alpha}^{\beta}\left(\left|\mathcal{K}^{\prime \prime}\right|^{q}, \eta\right)
\end{aligned}
$$

and

$$
\begin{aligned}
\left|\mathcal{K}^{\prime \prime}(\tau \beta+(1-\tau) \alpha)\right|^{q} & \leq \max \left\{\left|\mathcal{K}^{\prime \prime}(\alpha)\right|^{q},\left|\mathcal{K}^{\prime \prime}(\alpha)\right|^{q}+\eta\left(\left|\mathcal{K}^{\prime \prime}(\beta)\right|^{q},\left|\mathcal{K}^{\prime \prime}(\alpha)\right|^{q}\right)\right\} \\
& =: \mathbf{N}_{\alpha}^{\beta}\left(\left|\mathcal{K}^{\prime \prime}\right|^{q}, \eta\right) .
\end{aligned}
$$

Also, it is easy to see that

$$
\int_{0}^{1}(\tau+1)^{2 p} d \tau=\frac{2^{2 p+1}-1}{2 p+1}
$$

Using Lemma 10, Hölder's inequality, and thereafter Eqs. (12)-(14), one obtains

$$
\begin{aligned}
& \left|\int_{\alpha}^{\beta} \mathcal{K}(\mathfrak{r}) d \mathfrak{r}-\frac{1}{2}(\beta-\alpha)(\mathcal{K}(\alpha)+\mathcal{K}(\beta))+\frac{5}{4}(\beta-\alpha)^{2}\left(\mathcal{K}^{\prime}(\beta)-\mathcal{K}^{\prime}(\alpha)\right)\right| \\
& \leq \leq \frac{(\beta-\alpha)^{3}}{4}\left[\int_{0}^{1}(\tau+1)^{2}\left|\mathcal{K}^{\prime \prime}(\tau \alpha+(1-\tau) \beta)\right| d \tau\right. \\
& \left.\quad+\int_{0}^{1}(\tau+1)^{2}\left|\mathcal{K}^{\prime \prime}(\tau \beta+(1-\tau) \alpha)\right| d \tau\right] \\
& \leq \frac{(\beta-\alpha)^{3}}{4}\left[\left(\int_{0}^{1}(\tau+1)^{2 p} d \tau\right)^{\frac{1}{p}}\left(\int_{0}^{1}\left|\mathcal{K}^{\prime \prime}(\tau \alpha+(1-\tau) \beta)\right|^{q} d \tau\right)^{\frac{1}{q}}\right. \\
& \left.\quad+\left(\int_{0}^{1}(\tau+1)^{2 p} d \tau\right)^{\frac{1}{p}}\left(\int_{0}^{1}\left|\mathcal{K}^{\prime \prime}(\tau \beta+(1-\tau) \alpha)\right|^{q} d \tau\right)^{\frac{1}{q}}\right] \\
& \leq \frac{(\beta-\alpha)^{3}}{4}\left(\frac{2^{2 p+1}-1}{2 p+1}\right)^{\frac{1}{p}}\left[\left(\mathbf{M}_{\alpha}^{\beta}\left(\left|\mathcal{K}^{\prime \prime}\right|^{q}, \eta\right)\right)^{\frac{1}{q}}+\left(\mathbf{N}_{\alpha}^{\beta}\left(\left|\mathcal{K}^{\prime \prime}\right|^{q}, \eta\right)\right)^{\frac{1}{q}}\right] .
\end{aligned}
$$

That establishes the desired inequality.

Theorem 14 Let $\mathcal{K}: \mathfrak{D} \subset[0, \infty) \rightarrow \mathbb{R}$ be a differentiable function on $\mathfrak{D}^{\circ}$ such that $\mathcal{K}^{\prime \prime} \in$ $L([\alpha, \beta])$, where $\alpha, \beta \in \mathfrak{D}$ with $\alpha<\beta$. If $\left|\mathcal{K}^{\prime \prime}\right|^{q}$ is an $\eta$-quasiconvex function on $[\alpha, \beta]$ for 
$q \geq 1$, then

$$
\begin{aligned}
& \left|\int_{\alpha}^{\beta} \mathcal{K}(\mathfrak{r}) d \mathfrak{r}-\frac{1}{2}(\beta-\alpha)(\mathcal{K}(\alpha)+\mathcal{K}(\beta))+\frac{5}{4}(\beta-\alpha)^{2}\left(\mathcal{K}^{\prime}(\beta)-\mathcal{K}^{\prime}(\alpha)\right)\right| \\
& \quad \leq \frac{7(\beta-\alpha)^{3}}{12}\left[\left(\mathbf{M}_{\alpha}^{\beta}\left(\left|\mathcal{K}^{\prime \prime}\right|^{q}, \eta\right)\right)^{\frac{1}{q}}+\left(\mathbf{N}_{\alpha}^{\beta}\left(\left|\mathcal{K}^{\prime \prime}\right|^{q}, \eta\right)\right)^{\frac{1}{q}}\right]
\end{aligned}
$$

where $\mathbf{M}_{\alpha}^{\beta}\left(\left|\mathcal{K}^{\prime \prime}\right|^{q}, \eta\right)$ and $\mathbf{N}_{\alpha}^{\beta}\left(\left|\mathcal{K}^{\prime \prime}\right|^{q}, \eta\right)$ are defined as in Theorem 13.

Proof Applying Lemma 10 and the power integral inequality, one gets

$$
\begin{aligned}
\mid \int_{\alpha}^{\beta} & \mathcal{K}(\mathfrak{r}) d \mathfrak{r}-\frac{1}{2}(\beta-\alpha)(\mathcal{K}(\alpha)+\mathcal{K}(\beta))+\frac{5}{4}(\beta-\alpha)^{2}\left(\mathcal{K}^{\prime}(\beta)-\mathcal{K}^{\prime}(\alpha)\right) \mid \\
\leq & \frac{(\beta-\alpha)^{3}}{4}\left[\int_{0}^{1}(\tau+1)^{2}\left|\mathcal{K}^{\prime \prime}(\tau \alpha+(1-\tau) \beta)\right| d \tau\right. \\
& \left.+\int_{0}^{1}(\tau+1)^{2}\left|\mathcal{K}^{\prime \prime}(\tau \beta+(1-\tau) \alpha)\right| d \tau\right] \\
\leq & \frac{(\beta-\alpha)^{3}}{4}\left(\int_{0}^{1}(\tau+1)^{2} d \tau\right)^{1-\frac{1}{q}}\left[\left(\int_{0}^{1}(\tau+1)^{2} \mathbf{M}_{\alpha}^{\beta}\left(\left|\mathcal{K}^{\prime \prime}\right|^{q}, \eta\right) d \tau\right)^{\frac{1}{q}}\right. \\
& \left.+\left(\int_{0}^{1}(\tau+1)^{2} \mathbf{N}_{\alpha}^{\beta}\left(\left|\mathcal{K}^{\prime \prime}\right|^{q}, \eta\right) d \tau\right)^{\frac{1}{q}}\right] \\
\leq & \frac{(\beta-\alpha)^{3}}{4} \int_{0}^{1}(\tau+1)^{2} d \tau\left[\left(\mathbf{M}_{\alpha}^{\beta}\left(\left|\mathcal{K}^{\prime \prime}\right|^{q}, \eta\right)\right)^{\frac{1}{q}}+\left(\mathbf{N}_{\alpha}^{\beta}\left(\left|\mathcal{K}^{\prime \prime}\right|^{q}, \eta\right)\right)^{\frac{1}{q}}\right] \\
\leq & \frac{7(\beta-\alpha)^{3}}{12}\left[\left(\mathbf{M}_{\alpha}^{\beta}\left(\left|\mathcal{K}^{\prime \prime}\right|^{q}, \eta\right)\right)^{\frac{1}{q}}+\left(\mathbf{N}_{\alpha}^{\beta}\left(\left|\mathcal{K}^{\prime \prime}\right|^{q}, \eta\right)\right)^{\frac{1}{q}}\right] .
\end{aligned}
$$

Hence, the intended result is obtained.

\subsection{More inequalities via strong $\boldsymbol{\eta}$-quasiconvexity}

We now present some new estimates for $\int_{\alpha}^{\beta}(\mathfrak{r}-\alpha)^{p}(\beta-\mathfrak{r})^{q} \mathcal{K}(\mathfrak{r}) d \mathfrak{r}$ by means of the strong $\eta$-quasiconvexity.

Theorem 15 Let $\mathcal{K}:[\alpha, \beta] \subset[0, \infty) \rightarrow \mathbb{R}$ be continuous on $[\alpha, \beta]$ such that $\mathcal{K} \in L([\alpha, \beta])$ with $\alpha<\beta$. If $\mathcal{K}$ is strongly $\eta$-quasiconvex with nonnegative modulus $\mu$ on $[\alpha, \beta]$, then, for some fixed $p, q>0$, we obtain

$$
\begin{aligned}
& \int_{\alpha}^{\beta}(\mathfrak{r}-\alpha)^{p}(\beta-\mathfrak{r})^{q} \mathcal{K}(\mathfrak{r}) d \mathfrak{r} \\
& \quad \leq(\beta-\alpha)^{p+q+1}\left[\mathbf{M}_{\alpha}^{\beta}(\mathcal{K}, \eta) \mathcal{B}(p+1, q+1)-\mu(\beta-\alpha)^{2} \mathcal{B}(p+2, q+2)\right]
\end{aligned}
$$

Proof The strong $\eta$-quasiconvexity of $\mathcal{K}$ on $[\alpha, \beta]$ implies that, for $\tau \in[\alpha, \beta]$, one obtains

$$
\begin{aligned}
\mathcal{K}(\tau \alpha+(1-\tau) \beta) \leq & \max \{\mathcal{K}(\beta), \mathcal{K}(\beta)+\eta(\mathcal{K}(\alpha), \mathcal{K}(\beta))\} \\
& -\mu \tau(1-\tau)(\beta-\alpha)^{2} \\
= & : \mathbf{M}_{\alpha}^{\beta}(\mathcal{K}, \eta)-\mu \tau(1-\tau)(\beta-\alpha)^{2}
\end{aligned}
$$


for $\tau \in[0,1]$. From Lemma 11 and (16), we get

$$
\begin{aligned}
& \int_{\alpha}^{\beta}(\mathfrak{r}-\alpha)^{p}(\beta-\mathfrak{r})^{q} \mathcal{K}(\mathfrak{r}) d \mathfrak{r} \\
& \quad=(\beta-\alpha)^{p+q+1} \int_{0}^{1}(1-\tau)^{p} \tau^{q} \mathcal{K}(\tau \alpha+(1-\tau) \beta) d \tau \\
& \quad \leq(\beta-\alpha)^{p+q+1} \int_{0}^{1}(1-\tau)^{p} \tau^{q}\left[\mathbf{M}_{\alpha}^{\beta}(\mathcal{K}, \eta)-\mu \tau(1-\tau)(\beta-\alpha)^{2}\right] d \tau \\
& \quad \leq(\beta-\alpha)^{p+q+1}\left[\mathbf{M}_{\alpha}^{\beta}(\mathcal{K}, \eta) \int_{0}^{1}(1-\tau)^{p} \tau^{q} d \tau-\mu(\beta-\alpha)^{2} \int_{0}^{1} \tau^{q+1}(1-\tau)^{p+1} d \tau\right] \\
& \quad \leq(\beta-\alpha)^{p+q+1}\left[\mathbf{M}_{\alpha}^{\beta}(\mathcal{K}, \eta) \mathcal{B}(p+1, q+1)-\mu(\beta-\alpha)^{2} \mathcal{B}(p+2, q+2)\right],
\end{aligned}
$$

which gives the intended inequality.

Remark 16 Let $\eta(x, y)=x-y$. If, in addition, we assume that $\mathcal{K}$ in Theorem 15 is

1. increasing, then (15) boils down to

$$
\begin{aligned}
& \int_{\alpha}^{\beta}(\mathfrak{r}-\alpha)^{p}(\beta-\mathfrak{r})^{q} \mathcal{K}(\mathfrak{r}) d \mathfrak{r} \\
& \quad \leq(\beta-\alpha)^{p+q+1}\left[\mathcal{B}(p+1, q+1) \mathcal{K}(\beta)-\mu(\beta-\alpha)^{2} \mathcal{B}(p+2, q+2)\right]
\end{aligned}
$$

2. decreasing, then (15) becomes

$$
\begin{aligned}
& \int_{\alpha}^{\beta}(\mathfrak{r}-\alpha)^{p}(\beta-\mathfrak{r})^{q} \mathcal{K}(\mathfrak{r}) d \mathfrak{r} \\
& \quad \leq(\beta-\alpha)^{p+q+1}\left[\mathcal{B}(p+1, q+1) \mathcal{K}(\alpha)-\mu(\beta-\alpha)^{2} \mathcal{B}(p+2, q+2)\right] .
\end{aligned}
$$

Theorem 17 Let $\mathcal{K}:[\alpha, \beta] \subset[0, \infty) \rightarrow \mathbb{R}$ be continuous on $[\alpha, \beta]$ such that $\mathcal{K} \in L([\alpha, \beta])$ with $\alpha<\beta$, and let $x>1$ with $1 / x+1 / y=1$. If $|\mathcal{K}|^{y}$ is strongly $\eta$-quasiconvex with nonnegative modulus $\mu$ on $[\alpha, \beta]$, then, for some fixed $p, q>0$, we obtain

$$
\begin{aligned}
& \int_{\alpha}^{\beta}(\mathfrak{r}-\alpha)^{p}(\beta-\mathfrak{r})^{q} \mathcal{K}(\mathfrak{r}) d \mathfrak{r} \\
& \quad \leq(\beta-\alpha)^{p+q+1}[\mathcal{B}(p x+1, q x+1)]^{\frac{1}{x}}\left[\mathbf{M}_{\alpha}^{\beta}\left(|\mathcal{K}|^{y}, \eta\right)-\mu \frac{(\beta-\alpha)^{2}}{6}\right]^{\frac{1}{y}}
\end{aligned}
$$

Proof The strong $\eta$-quasiconvexity of the function $|\mathcal{K}|^{y}$ on $[\alpha, \beta]$ implies that, for $\tau \in$ $[0,1]$,

$$
\begin{aligned}
|\mathcal{K}(\tau \alpha+(1-\tau) \beta)|^{y} \leq & \max \left\{|\mathcal{K}(\beta)|^{y},|\mathcal{K}(\beta)|^{y}+\eta\left(|\mathcal{K}(\alpha)|^{y},|\mathcal{K}(\beta)|^{y}\right)\right\} \\
& -\mu \tau(1-\tau)(\beta-\alpha)^{2} \\
= & : \mathbf{M}_{\alpha}^{\beta}\left(|\mathcal{K}|^{y}, \eta\right)-\mu \tau(1-\tau)(\beta-\alpha)^{2} .
\end{aligned}
$$


Applying (18) to Lemma 11, Hölder's inequality and the definition of the Beta function given above, one obtains

$$
\begin{aligned}
& \int_{\alpha}^{\beta}(\mathfrak{r}-\alpha)^{p}(\beta-\mathfrak{r})^{q} \mathcal{K}(\mathfrak{r}) d \mathfrak{r} \\
& \quad \leq(\beta-\alpha)^{p+q+1} \int_{0}^{1}(1-\tau)^{p} \tau^{q}|\mathcal{K}(\tau \alpha+(1-\tau) \beta)| d \tau \\
& \quad \leq(\beta-\alpha)^{p+q+1}\left[\int_{0}^{1}(1-\tau)^{p x} \tau^{q x} d \tau\right]^{\frac{1}{x}}\left[\int_{0}^{1}|\mathcal{K}(\tau \alpha+(1-\tau) \beta)|^{y} d \tau\right]^{\frac{1}{y}} \\
& \quad \leq(\beta-\alpha)^{p+q+1}\left[\int_{0}^{1}(1-\tau)^{p x} \tau^{q x} d \tau\right]^{\frac{1}{x}}\left[\int_{0}^{1}\left(\mathbf{M}_{\alpha}^{\beta}\left(|\mathcal{K}|^{y}, \eta\right)-\mu \tau(1-\tau)(\beta-\alpha)^{2}\right) d \tau\right]^{\frac{1}{y}} \\
& \quad=(\beta-\alpha)^{p+q+1}[\mathcal{B}(p x+1, q x+1)]^{\frac{1}{x}}\left[\mathbf{M}_{\alpha}^{\beta}\left(|\mathcal{K}|^{y}, \eta\right)-\mu \frac{(\beta-\alpha)^{2}}{6}\right]^{\frac{1}{y}} .
\end{aligned}
$$

That completes the proof.

We end this section with our last result.

Theorem 18 Let $\mathcal{K}:[\alpha, \beta] \subset[0, \infty) \rightarrow \mathbb{R}$ be continuous on $[\alpha, \beta]$ such that $\mathcal{K} \in L([\alpha, \beta])$ with $\alpha<\beta$, and let $s \geq 1$. If $|\mathcal{K}|^{s}$ is strongly $\eta$-quasiconvex with nonnegative modulus $\mu$ on $[\alpha, \beta]$, then, for some fixed $p, q>0$, we obtain

$$
\begin{aligned}
\int_{\alpha}^{\beta}(\mathfrak{r}-\alpha)^{p}(\beta-\mathfrak{r})^{q} \mathcal{K}(\mathfrak{r}) d \mathfrak{r} \\
\quad \leq(\beta-\alpha)^{p+q+1}[\mathcal{B}(p+1, q+1)]^{\frac{s-1}{s}} \\
\quad \times\left[\mathcal{B}(p+1, q+1) \mathbf{M}_{\alpha}^{\beta}\left(|\mathcal{K}|^{s}, \eta\right)-\mu(\beta-\alpha)^{2} \mathcal{B}(p+2, q+2)\right]^{\frac{1}{s}}
\end{aligned}
$$

Proof We proceed as in the proof of Theorem 17, but this time we use the mean power inequality. We have

$$
\begin{aligned}
& \int_{\alpha}^{\beta}(\mathfrak{r}-\alpha)^{p}(\beta-\mathfrak{r})^{q} \mathcal{K}(\mathfrak{r}) d \mathfrak{r} \\
& \leq(\beta-\alpha)^{p+q+1} \int_{0}^{1}\left[(1-\tau)^{p} \tau^{q}\right]^{\frac{s-1}{s}}\left[(1-\tau)^{p} \tau^{q}\right]^{\frac{1}{s}}|\mathcal{K}(\tau \alpha+(1-\tau) \beta)| d \tau \\
& \leq(\beta-\alpha)^{p+q+1}\left[\int_{0}^{1}(1-\tau)^{p} \tau^{q} d \tau\right]^{\frac{s-1}{s}}\left[\int_{0}^{1}(1-\tau)^{p} \tau^{q}|\mathcal{K}(\tau \alpha+(1-\tau) \beta)|^{s} d \tau\right]^{\frac{1}{s}} \\
& \leq(\beta-\alpha)^{p+q+1}\left[\int_{0}^{1}(1-\tau)^{p} \tau^{q} d \tau\right]^{\frac{s-1}{s}} \\
& \times\left[\int_{0}^{1}(1-\tau)^{p} \tau^{q}\left(\mathbf{M}_{\alpha}^{\beta}\left(|\mathcal{K}|^{s}, \eta\right)-\mu \tau(1-\tau)(\beta-\alpha)^{2}\right) d \tau\right]^{\frac{1}{s}} \\
&=(\beta-\alpha)^{p+q+1}[\mathcal{B}(p+1, q+1)]^{\frac{s-1}{s}} \\
& \times\left[\mathcal{B}(p+1, q+1) \mathbf{M}_{\alpha}^{\beta}\left(|\mathcal{K}|^{s}, \eta\right)-\mu(\beta-\alpha)^{2} \mathcal{B}(p+2, q+2)\right]^{\frac{1}{s}},
\end{aligned}
$$

which gives the desired result. 
Remark 19 Similar corollaries, as stated in Remark 16, can be deduced from Theorems 17 and 18. Moreover, by taking $\eta(x, y)=x-y$ and $\mu=0$, our Theorem 15 becomes Theorem 5 of Özdemir et al., and Theorems 17 and 18 reduce to Theorems 6 and 7 (due to Liu), respectively.

\section{Applications to trapezoidal formula}

We start by recalling the following well-known result: let $\mathcal{K}:[\alpha, \beta] \rightarrow \mathbb{R}$ be a function whose second derivative exists in $(\alpha, \beta)$ and let $\mathbf{P}$ be a partition of the interval $[\alpha, \beta]$, that is, $\mathbf{P}: \alpha=\mathfrak{r}_{1}<\mathfrak{r}_{2}<\cdots<\mathfrak{r}_{m-1}<\mathfrak{r}_{m}=\beta$, then

$$
\mathfrak{J}:=\int_{\alpha}^{\beta} \mathcal{K}(\mathfrak{r}) d \mathfrak{r}=\mathbb{F}(\mathcal{K}, \mathbf{P})+\mathbb{E}(\mathcal{K}, \mathbf{P}),
$$

where the trapezoidal formula $\mathbb{F}(\mathcal{K}, \mathbf{P})$ is defined by

$$
\mathbb{F}(\mathcal{K}, \mathbf{P})=\sum_{k=0}^{m-1} \frac{\mathcal{K}\left(\mathfrak{r}_{k}\right)+\mathcal{K}\left(\mathfrak{r}_{k+1}\right)}{2}\left(\mathfrak{r}_{k+1}-\mathfrak{r}_{k}\right)
$$

and the approximation error $\mathbb{E}(\mathcal{K}, \mathbf{P})$ of the integral $\mathfrak{J}$ by the trapezoidal formula $\mathbb{F}(\mathcal{K}, \mathbf{P})$ and satisfies the inequality

$$
|\mathbb{E}(\mathcal{K}, \mathbf{P})| \leq \frac{Q}{12} \sum_{k=0}^{m-1}\left(\mathfrak{r}_{k+1}-\mathfrak{r}_{k}\right)^{3}
$$

with $Q=\max _{\mathfrak{r} \in(\alpha, \beta)}\left|\mathcal{K}^{\prime \prime}(\mathfrak{r})\right|<\infty$. In this section, we establish some perturbed new inequalities analogous to the estimate in (20) for twice differentiable functions.

Proposition 20 Let $\mathcal{K}: \mathfrak{D} \subset[0, \infty) \rightarrow \mathbb{R}$ be a differentiable function on $\mathfrak{D}^{\circ}$ such that $\mathcal{K}^{\prime \prime} \in$ $L([\alpha, \beta])$, where $\alpha, \beta \in \mathfrak{D}$ with $\alpha<\beta$.If $\left|\mathcal{K}^{\prime \prime}\right|$ is quasiconvex on $[\alpha, \beta]$, then for every partition $\mathbf{P}$ of $[\alpha, \beta]$

$$
|\mathbb{E}(\mathcal{K}, \mathbf{P})+5 \mathbb{G}(\mathcal{K}, \mathbf{P})| \leq \frac{7}{6} \sum_{k=0}^{m-1}\left(\mathfrak{r}_{k+1}-\mathfrak{r}_{k}\right)^{3} \max \left\{\left|\mathcal{K}^{\prime \prime}\left(\mathfrak{r}_{k}\right)\right|,\left|\mathcal{K}^{\prime \prime}\left(\mathfrak{r}_{k+1}\right)\right|\right\},
$$

where $\mathbb{G}(\mathcal{K}, \mathbf{P})=\sum_{k=0}^{m-1}\left(\mathfrak{r}_{k+1}-\mathfrak{r}_{k}\right)^{2} \frac{\mathcal{K}^{\prime}\left(\mathfrak{r}_{k+1}\right)-\mathcal{K}^{\prime}\left(\mathfrak{r}_{k}\right)}{4}$.

Proof By using the function $\eta(x, y)=x-y$ in (9) of Theorem 12, one gets

$$
\begin{aligned}
& \left|\int_{\alpha}^{\beta} \mathcal{K}(\mathfrak{r}) d \mathfrak{r}-\frac{1}{2}(\beta-\alpha)(\mathcal{K}(\alpha)+\mathcal{K}(\beta))+\frac{5}{4}(\beta-\alpha)^{2}\left(\mathcal{K}^{\prime}(\beta)-\mathcal{K}^{\prime}(\alpha)\right)\right| \\
& \quad \leq \frac{7(\beta-\alpha)^{3}}{6} \max \left\{\left|\mathcal{K}^{\prime \prime}(\alpha)\right|,\left|\mathcal{K}^{\prime \prime}(\beta)\right|\right\} .
\end{aligned}
$$

Now employing (21) for the subintervals $\left[\mathfrak{r}_{k}, \mathfrak{r}_{k+1}\right]$ for $k=\overline{0, m-1}$ of $\mathbf{P}$, we obtain

$$
\begin{aligned}
& \left|\int_{\mathfrak{r}_{k}}^{\mathfrak{r}_{k+1}} \mathcal{K}(\mathfrak{r}) d \mathfrak{r}-\frac{1}{2}\left(\mathfrak{r}_{k+1}-\mathfrak{r}_{k}\right)\left(\mathcal{K}\left(\mathfrak{r}_{k}\right)+\mathcal{K}\left(\mathfrak{r}_{k+1}\right)\right)+\frac{5}{4}\left(\mathfrak{r}_{k+1}-\mathfrak{r}_{k}\right)^{2}\left(\mathcal{K}^{\prime}\left(\mathfrak{r}_{k+1}\right)-\mathcal{K}^{\prime}\left(\mathfrak{r}_{k}\right)\right)\right| \\
& \quad \leq \frac{7\left(\mathfrak{r}_{k+1}-\mathfrak{r}_{k}\right)^{3}}{6} \max \left\{\left|\mathcal{K}^{\prime \prime}\left(\mathfrak{r}_{k}\right)\right|,\left|\mathcal{K}^{\prime \prime}\left(\mathfrak{r}_{k+1}\right)\right|\right\} .
\end{aligned}
$$


Summing both sides of the above inequality over $k$ from 0 to $m-1$ amounts to

$$
\begin{aligned}
& \mid \sum_{k=0}^{m-1} \int_{\mathfrak{r}_{k}}^{\mathfrak{r}_{k+1}} \mathcal{K}(\mathfrak{r}) d \mathfrak{r}-\sum_{k=0}^{m-1}\left(\mathfrak{r}_{k+1}-\mathfrak{r}_{k}\right) \frac{\mathcal{K}\left(\mathfrak{r}_{k}\right)+\mathcal{K}\left(\mathfrak{r}_{k+1}\right)}{2} \\
& \quad+5 \sum_{k=0}^{m-1}\left(\mathfrak{r}_{k+1}-\mathfrak{r}_{k}\right)^{2} \frac{\mathcal{K}^{\prime}\left(\mathfrak{r}_{k+1}\right)-\mathcal{K}^{\prime}\left(\mathfrak{r}_{k}\right)}{4} \mid \\
& \leq \frac{7}{6} \sum_{k=0}^{m-1}\left(\mathfrak{r}_{k+1}-\mathfrak{r}_{k}\right)^{3} \max \left\{\left|\mathcal{K}^{\prime \prime}\left(\mathfrak{r}_{k}\right)\right|,\left|\mathcal{K}^{\prime \prime}\left(\mathfrak{r}_{k+1}\right)\right|\right\}
\end{aligned}
$$

from which the desired inequality is obtained.

By applying Proposition 20, the following consequence is obtained.

Corollary 21 Let $\mathcal{K}: \mathfrak{D} \subset[0, \infty) \rightarrow \mathbb{R}$ be a differentiable function on $\mathfrak{D}^{\circ}$ such that $\mathcal{K}^{\prime \prime} \in$ $L([\alpha, \beta])$, where $\alpha, \beta \in \mathfrak{D}$ with $\alpha<\beta$. If $\left|\mathcal{K}^{\prime \prime}\right|$ is quasiconvex on $[\alpha, \beta]$, then, for every partition $\mathbf{P}$ of $[\alpha, \beta]$, we have:

I. If $\left|\mathcal{K}^{\prime \prime}\right|$ is increasing, then

$$
|\mathbb{E}(\mathcal{K}, \mathbf{P})+5 \mathbb{G}(\mathcal{K}, \mathbf{P})| \leq \frac{7}{6} \sum_{k=0}^{m-1}\left(\mathfrak{r}_{k+1}-\mathfrak{r}_{k}\right)^{3}\left|\mathcal{K}^{\prime \prime}\left(\mathfrak{r}_{k+1}\right)\right| .
$$

II. If $\left|\mathcal{K}^{\prime \prime}\right|$ is decreasing, then

$$
|\mathbb{E}(\mathcal{K}, \mathbf{P})+5 \mathbb{G}(\mathcal{K}, \mathbf{P})| \leq \frac{7}{6} \sum_{k=0}^{m-1}\left(\mathfrak{r}_{k+1}-\mathfrak{r}_{k}\right)^{3}\left|\mathcal{K}^{\prime \prime}\left(\mathfrak{r}_{k}\right)\right| .
$$

Proposition 22 Let $\mathcal{K}: \mathfrak{D} \subset[0, \infty) \rightarrow \mathbb{R}$ be a differentiable function on $\mathfrak{D}^{\circ}$ such that $\mathcal{K}^{\prime \prime} \in L([\alpha, \beta])$, where $\alpha, \beta \in \mathfrak{D}$ with $\alpha<\beta$, and let $p>1$ with $1 / p+1 / q=1$. If $\left|\mathcal{K}^{\prime \prime}\right|^{q}$ is a quasiconvex function on $[\alpha, \beta]$, then for every partition $\mathbf{P}$ of $[\alpha, \beta]$

$$
\begin{aligned}
& |\mathbb{E}(\mathcal{K}, \mathbf{P})+5 \mathbb{G}(\mathcal{K}, \mathbf{P})| \\
& \quad \leq \frac{1}{2}\left(\frac{2^{2 p+1}-1}{2 p+1}\right)^{\frac{1}{p}} \sum_{k=0}^{m-1}\left(\mathfrak{r}_{k+1}-\mathfrak{r}_{k}\right)^{3}\left[\max \left\{\left|\mathcal{K}^{\prime \prime}\left(\mathfrak{r}_{k}\right)\right|^{q},\left|\mathcal{K}^{\prime \prime}\left(\mathfrak{r}_{k+1}\right)\right|^{q}\right\}\right]^{\frac{1}{q}} .
\end{aligned}
$$

Proof We apply Theorem 13 to the bifunction $\eta(x, y)=x-y$, and we proceed as in the proof of Proposition 20.

Now applying Proposition 22, one obtains the following corollary.

Corollary 23 Let $\mathcal{K}: \mathfrak{D} \subset[0, \infty) \rightarrow \mathbb{R}$ be a differentiable function on $\mathfrak{D}^{\circ}$ such that $\mathcal{K}^{\prime \prime} \in$ $L([\alpha, \beta])$, where $\alpha, \beta \in \mathfrak{D}$ with $\alpha<\beta$, and let $p>1$ with $1 / p+1 / q=1$. If $\left|\mathcal{K}^{\prime \prime}\right|^{q}$ is a quasiconvex function on $[\alpha, \beta]$, then, for every partition $\mathbf{P}$ of $[\alpha, \beta]$, we get:

I. If $\left|\mathcal{K}^{\prime \prime}\right|^{q}$ is increasing, then

$$
|\mathbb{E}(\mathcal{K}, \mathbf{P})+5 \mathbb{G}(\mathcal{K}, \mathbf{P})| \leq \frac{1}{2}\left(\frac{2^{2 p+1}-1}{2 p+1}\right)^{\frac{1}{p}} \sum_{k=0}^{m-1}\left(\mathfrak{r}_{k+1}-\mathfrak{r}_{k}\right)^{3}\left|\mathcal{K}^{\prime \prime}\left(\mathfrak{r}_{k+1}\right)\right| .
$$


II. If $\left|\mathcal{K}^{\prime \prime}\right|^{q}$ is decreasing, then

$$
|\mathbb{E}(\mathcal{K}, \mathbf{P})+5 \mathbb{G}(\mathcal{K}, \mathbf{P})| \leq \frac{1}{2}\left(\frac{2^{2 p+1}-1}{2 p+1}\right)^{\frac{1}{p}} \sum_{k=0}^{m-1}\left(\mathfrak{r}_{k+1}-\mathfrak{r}_{k}\right)^{3}\left|\mathcal{K}^{\prime \prime}\left(\mathfrak{r}_{k}\right)\right| .
$$

Employing Theorem 14, we conclude this section by presenting a generalization of Proposition 20.

Proposition 24 Let $\mathcal{K}: \mathfrak{D} \subset[0, \infty) \rightarrow \mathbb{R}$ be a differentiable function on $\mathfrak{D}^{\circ}$ such that $\mathcal{K}^{\prime \prime} \in$ $L([\alpha, \beta])$, where $\alpha, \beta \in \mathfrak{D}$ with $\alpha<\beta$. If $\left|\mathcal{K}^{\prime \prime}\right|^{q}$ is a quasiconvex function on $[\alpha, \beta]$ for $q \geq 1$, then for every partition $\mathbf{P}$ of $[\alpha, \beta]$

$$
|\mathbb{E}(\mathcal{K}, \mathbf{P})+5 \mathbb{G}(\mathcal{K}, \mathbf{P})| \leq \frac{7}{6} \sum_{k=0}^{m-1}\left(\mathfrak{r}_{k+1}-\mathfrak{r}_{k}\right)^{3}\left[\max \left\{\left|\mathcal{K}^{\prime \prime}\left(\mathfrak{r}_{k}\right)\right|^{q},\left|\mathcal{K}^{\prime \prime}\left(\mathfrak{r}_{k+1}\right)\right|^{q}\right\}\right]^{\frac{1}{q}}
$$

\section{Applications to special means}

In this section, we apply some of our theorems to the following special means for distinct real numbers $x$ and $y$.

1. Arithmetic mean:

$$
\mathcal{A}(x, y)=\frac{x+y}{2} .
$$

2. Geometric mean:

$$
\mathcal{G}(x, y)=\sqrt{x y}, \quad x, y>0 .
$$

3. Harmonic mean:

$$
\mathcal{H}(x, y)=\frac{2 x y}{x+y} .
$$

4. Logarithmic mean:

$$
\mathcal{L}(x, y)=\frac{x-y}{\ln |x|-\ln |y|}, \quad|x| \neq|y|, \text { and } x, y \neq 0 .
$$

5. Generalized logarithmic mean:

$$
\mathcal{L}_{m}(x, y)=\left[\frac{y^{m+1}-x^{m+1}}{(m+1)(y-x)}\right]^{\frac{1}{m}}, \quad m \in \mathbb{N} .
$$

Proposition 25 Suppose $\alpha, \beta \in \mathbb{R}$ with $\alpha<\beta$ and $m \in \mathbb{N}, m \geq 2$. Then we get

$$
\begin{aligned}
& \left|\mathcal{L}_{m}^{m}(\alpha, \beta)-\mathcal{A}\left(\alpha^{m}, \beta^{m}\right)+\frac{5}{4} m(\beta-\alpha)\left(\beta^{m-1}-\alpha^{m-1}\right)\right| \\
& \leq \frac{7}{6} m(m-1)(\beta-\alpha)^{2} \max \left\{|\alpha|^{m-2},|\beta|^{m-2}\right\} .
\end{aligned}
$$


Proof Let $\eta(s, t)=s-t$ and $\mathcal{K}(s)=s^{m}, s \in \mathbb{R}$. The absolute value of the function $\mathcal{K}^{\prime \prime}(s)=$ $m(m-1) s^{m-2}, s \in \mathbb{R}$ is $\eta$-quasiconvex with respect to the bifunction $\eta$. Now, applying Theorem 12 to $\mathcal{K}$ and $\eta$, one obtains (22).

Proposition 26 Suppose $\alpha, \beta \in \mathbb{R}$ with $\alpha<\beta$ and $m \in \mathbb{N}, m \geq 2$. Then, for $p, q>1$ with $1 / p+1 / q=1$, we get

$$
\begin{aligned}
& \left|\mathcal{L}_{m}^{m}(\alpha, \beta)-\mathcal{A}\left(\alpha^{m}, \beta^{m}\right)+\frac{5}{4} m(\beta-\alpha)\left(\beta^{m-1}-\alpha^{m-1}\right)\right| \\
& \quad \leq \frac{(\beta-\alpha)^{2}}{2} m(m-1)\left(\frac{2^{2 p+1}-1}{2 p+1}\right)^{\frac{1}{p}}\left[\max \left\{|\alpha|^{q(m-2)},|\beta|^{q(m-2)}\right\}\right]^{\frac{1}{q}} .
\end{aligned}
$$

Proof We use Theorem 13 and proceed as in the proof of Proposition 25.

Proposition 27 Suppose $\alpha, \beta \in \mathbb{R}$ with $0<\alpha<\beta$. Then, for $q \geq 1$, we have

$$
\left|\mathcal{L}^{-1}(\alpha, \beta)-\mathcal{H}^{-1}(\alpha, \beta)+\frac{5}{2}(\beta-\alpha)^{2} \frac{\mathcal{A}(\alpha, \beta)}{\mathcal{G}^{4}(\alpha, \beta)}\right| \leq \frac{7}{6}(\beta-\alpha)^{2} \frac{2^{1 / q}}{\alpha^{3}} .
$$

Proof Here, we consider the function $\mathcal{K}(s)=1 / s, s \in[\alpha, \beta]$. The function $\left|\mathcal{K}^{\prime \prime}(s)\right|^{q}=\left(\frac{2}{s^{3}}\right)^{q}$ is $\eta$-quasiconvex with respect to $\eta(s, t)=s-t$. Employing Theorem 14 to $\mathcal{K}$ and $\eta$, we get the desired result.

\section{Conclusion}

Many integral inequalities associated with the $\eta$-quasiconvex and strongly $\eta$-quasiconvex functions have been established. Results obtained herein generalize, extend and refine some published theorems in the literature. Applications to the trapezoidal formula and some special means are also considered. We anticipate that this work will trigger further investigation in this direction. Some new inequalities can also be found in $[3,9,10,12,14$, $15,20]$.

\section{Acknowledgements}

Many thanks go to the referees for their valuable comments and suggestions.

Funding

There is no funding to report at this point in time.

\section{Availability of data and materials}

Not applicable in this work.

Competing interests

The author declares that there are no competing interests.

Authors' contributions

The sole author contributed $100 \%$ to this article. All authors read and approved the final manuscript.

\section{Publisher's Note}

Springer Nature remains neutral with regard to jurisdictional claims in published maps and institutional affiliations. 


\section{References}

1. Alomari, M., Darus, M., Dragomir, S.S.: Inequalities of Hermite-Hadamard's type for functions whose derivatives absolute values are quasi-convex. RGMIA Res. Rep. Coll. 12(14), (2009)

2. Awan, M.U., Noorb, M.A., Noorb, K.I., Safdarb, F.: On strongly generalized convex functions. FILOMAT 31(18), 5783-5790 (2017)

3. Chu, Y.-M., Adil Khan, M., Ali, T., Dragomir, S.S.: Inequalities for $\alpha$-fractional differentiable functions. J. Inequal. Appl. 2017, 93 (2017)

4. Delavar, M.R., Dragomir, S.S.: On $\eta$-convexity. Math. Inequal. Appl. 20(1), 203-216 (2017)

5. Gordji, M.E., Dragomir, S.S., Delavar, M.R.: An inequality related to $\eta$-convex functions (II). Int. J Nonlinear Anal. Appl. 6(2), 26-32 (2015)

6. Gordji, M.E., Delavar, M.R., Sen, M.D.L.: On $\varphi$-convex functions. J. Math. Inequal. 10(1), 173-183 (2016)

7. Kermausuor, S., Nwaeze, E.R.: Some new inequalities involving the Katugampola fractional integrals for strongly $\eta$-convex functions. Tbilisi Math. J. 12(1), 117-130 (2019)

8. Kermausuor, S., Nwaeze, E.R., Tameru, A.M.: New integral inequalities via the Katugampola fractional integrals for functions whose second derivatives are strongly $\eta$-convex. Mathematics 7(2). Article ID 183 (2019)

9. Adil Khan, M., Begum, S., Khurshid, Y., Chu, Y.M.: Ostrowski type inequalities involving conformable fractional integrals. J. Inequal. Appl. 2018, 70 (2018)

10. Adil Khan, M., Khurshid, Y., Du, T., Chu, Y.-M.: Generalization of Hermite-Hadamard type inequalities via conformable fractional integrals. Journal of Function Spaces 2018, Article ID 5357463 (2018)

11. Ion, D.A.: Some estimates on the Hermite-Hadamard inequality through quasi-convex functions. Annals of University of Craiova, Math. Comp. Sci. Ser. 34, 82-87 (2007)

12. Iqbal, A., Adil Khan, M., Ullah, S., Kashuri, A., Chu, Y.-M.: Hermite-Hadamard type inequalities pertaining conformable fractional integrals and their applications. AIP advances 8(075101), 1-18 (2018)

13. Khan, M.A., Khurshid, Y., Ali, T.: Hermite-Hadamard inequality for fractional integrals via $\eta$-convex functions. Acta Math. Univ. Comenianae. LXXXVI(1), 153-164 (2017)

14. Adil Khan, M., Iqbal, A., Suleman, M., Chu, Y.-M.: Hermite-Hadamard type inequalities for fractional integrals via green function. J. Inequal. Appl. 2018, 161 (2018)

15. Khurshid, Y., Adil Khan, M., Chu, Y.-M.: Hermite-Hadamard-Fejer inequalities for conformable fractional integrals via preinvex functions. Journal of Function Spaces 2019, Article ID 3146210 (2019)

16. Liu, W.J.: New integral inequalities via $(\alpha, m)$-convexity and quasi-convexity. Hacet. J. Math. Stat. 42, 289-297 (2013)

17. Nwaeze, E.R., Torres, D.F.M.: New inequalities for $\eta$-quasiconvex functions. In: Anastassiou, G., Rassias, J. (eds.) Frontiers in Functional Equations and Analytic Inequalities (FEAI). Springer, New York. Accepted

18. Nwaeze, E.R., Torres, D.F.M.: Novel results on the Hermite-Hadamard kind inequality for $\eta$-convex functions by means of the ( $k, r)$-fractional integral operators. In: Dragomir, S.S., Agarwal, P., Jleli, M., Samet, B. (eds.) Advances in Mathematical Inequalities and Applications (AMIA). Trends in Mathematics, pp. 311-321. Birkhäuser, Singapore (2018)

19. Nwaeze, E.R.: Generalized fractional integral inequalities by means of quasiconvexity. Adv. Diff. Equ. 2019,262 (2019)

20. Nwaeze, E.R.: Inequalities of the Hermite-Hadamard type for quasi-convex functions via the ( $k, s)$-Riemann-Liouville fractional integrals. Fractional Differ. Calc. 8(2), 327-336 (2018)

21. Nwaeze, E.R., Kermausuor, S., Tameru, A.M.: Some new k-Riemann-Liouville fractional integral inequalities associated with the strongly $\eta$-quasiconvex functions with modulus $\mu \geq 0$. J. Inequal. Appl. 2018, 139 (2018)

22. Ozdemir, M.E., Set, E., Alomari, M.: Integral inequalities via several kinds of convexity. Creat. Math. Inform. 20(1), 62-73 (2011)

23. Tunç, M., Şanal, Ü.: Some perturbed trapezoid inequalities for convex, s-convex and tgs-convex functions and applications. Tbil. Math. J. 8(2), 87-102 (2015)

\section{Submit your manuscript to a SpringerOpen ${ }^{\circ}$ journal and benefit from:}

- Convenient online submission

- Rigorous peer review

- Open access: articles freely available online

- High visibility within the field

- Retaining the copyright to your article

Submit your next manuscript at $\boldsymbol{s p r i n g e r o p e n . c o m ~}$ 